\title{
Factors associated with typhoid relapse in the era of multiple drug resistant strains
}

\author{
Kaashif Aqeeb Ahmad ${ }^{1,3}$, Liaqat Hayat Khan ${ }^{2,3}$, Bakht Roshan ${ }^{3}$, Zulfiqar A. Bhutta ${ }^{3}$ \\ ${ }^{1}$ Pediatrix Medical Group, San Antonio, Texas, USA \\ ${ }^{2}$ Pediatrix Medical Group, Alexandria, Louisiana, USA \\ ${ }^{3}$ Division of Women and Child Health, The Aga Khan University Hospital, Karachi, Pakistan
}

\begin{abstract}
Introduction: Typhoid has an estimated global burden of greater than 27 million cases per annum with a clinical relapse rate of $5 \%$ to $20 \%$. Despite the large relapse burden, the factors associated with relapse are largely unknown.

Methodology: We have followed a protocol for the diagnosis and management of pediatric typhoid since 1988. We report factors associated with relapse of culture-proven enteric fever in 1,650 children presenting to the Aga Khan University Medical Center, Karachi, Pakistan, over a 15-year period.

Results: In those infected with multiple drug resistant (MDR) strains, factors associated with subsequent relapse include constipation at presentation and presentation within 14 days of fever onset. Diarrhoea in those children infected with drug sensitive strains had an association with decreased subsequent relapse, as was quinolone therapy.

Conclusions: Multiple clinical factors at presentation are associated with subsequent typhoid fever relapse. These factors may be postulated to be associated with subsequent relapse due to alterations in the reticuloendothelial system organism load. These data will be valuable in developing algorithms for clinical follow-up in children infected with MDR enteric fever.
\end{abstract}

Key words: child; typhoid fever; Pakistan; recurrence; Salmonella

J Infect Dev Ctries 2011; 5(10):727-731.

(Received 12 May 2010 - Accepted 24 March 2011)

Copyright (C) 2011 Ahmad et al. This is an open-access article distributed under the Creative Commons Attribution License, which permits unrestricted use, distribution, and reproduction in any medium, provided the original work is properly cited.

\section{Introduction}

With an estimated global burden of over 27 million cases and 200,000 deaths annually [1], typhoid fever causes substantial morbidity and mortality throughout the developing world. Salmonella enterica subsp. enterica serovar Typhi ( $S$. Typhi) and Salmonella enterica subsp. enterica serovar Paratyphi ( $S$. Paratyphi) are the causative agents of typhoid fever, which is known to have three clinical outcomes: cure, death, and a carrier state [2]. Those having a clinical cure may relapse in 5\% to $20 \%$ of cases within weeks to months, with recurrence of clinical disease that is usually milder than the initial illness [3].

The emergence of multi-drug resistant (MDR) strains of Salmonella causing typhoid fever has been well documented throughout the world since the early 1990s [4]. These strains are defined as those exhibiting plasmid mediated resistance to ampicillin, chloramphenicol, and trimethoprim-sulfamethoxazole and are known to cause increased morbidity and mortality [5]. Due to the substantial public health burden of typhoid fever it is important to recognize factors associated with an increased risk of relapse; however, little data is available. Such information would be useful in elaborating prognostic factors and in evolving algorithms for the follow-up management of children presenting with typhoid fever.

As part of our ongoing studies on typhoid fever at the Aga Khan University Medical Center (AKUMC) in Karachi, Pakistan, we reviewed the frequency of relapse and factors predicting it among our pediatric population. We hypothesized that the emergence of MDR typhoid fever would lead to a higher overall rate of relapse and that those patients infected with MDR strains who received quinolones or cephalosporins would be protected against relapse.

\section{Methodology}

The Aga Khan University Medical Center in Karachi, Pakistan (population 14 million), is a 400bed teaching hospital with active ambulatory care and pediatric services. The Department of Pediatrics has followed a specific protocol for the diagnosis and 
management of suspected typhoid fever in children since 1988 and a database of all culture proven cases of typhoid fever has been maintained. In all cases, after a detailed clinical evaluation a complete blood picture and blood culture were obtained. In addition, a bone marrow aspirate for culture was also performed in cases where the child had received potentially active antibiotics for $\geq 72$ hours prior to admission. The blood or bone marrow specimens were inoculated into two bottles containing brainheart infusion broth or thioglycollate broth respectively. Each culture bottle was examined daily and colonies were subcultured onto blood agar and MacConkey agar after 24, 48 and 72 hours. Nonlactose fermenting colonies from these were biochemically identified with API 20E strips (Analytab Products, Plainview, New York, USA) and typed for Salmonella $\mathrm{O}$ antigen by slide agglutination with specific antisera. Antibiotic susceptibility testing was performed by the Kirby-Bauer disc diffusion method [6]. For cost considerations only one blood culture was obtained in suspected cases and a stool examination and culture was restricted to cases presenting with diarrhoea. Other investigations such as urine cultures, liver function tests, serum electrolytes were performed as clinically indicated.

At the onset of this protocol, cases with suspected typhoid fever were empirically treated with oral chloramphenicol or ampicillin and therapy was subsequently adjusted according to sensitivity results. In the mid 1990s, due to concerns for the increasing trend of multidrug resistance among typhoidal isolates, our protocol was modified. Clinicians were then allowed, for children severely ill with toxicity or those who had failed to respond to first-line antibiotics administered prior to our evaluation, to hospitalize for suspicion of infection with MDR typhoid. These patients then received quinolones or IV cephalosporins. All patients received antibiotics for $\geq 14$ days depending upon the severity of illness and the response to the antibiotic used. Successful therapy was defined as recovery from clinical illness with defervescence (temperature $\leq 37.5^{\circ} \mathrm{C}$ for $\geq 48$ hours). Relapse was considered present with the recurrence of clinical disease, culture-proven infection with Salmonella Typhi and Paratyphi isolates (as described above), and with an antibiogram identical to the original isolates, within eight weeks of cessation of successful therapy of the initial infection.

Data was recorded on a specially designed pro forma and transferred to a computer database.
Comparative data on baseline characteristics, morbidity patterns, and outcome were analyzed by $\chi^{2}$ test and t-test, as needed, using SPSS (Windows version 11.0, SPSS Inc., Chicago, Illinois). A p-value of 0.05 was selected as the cutoff for significance.

The clinical protocol was approved by the Human Subjects Review Committee of the Aga Khan University.

\section{Results}

Over a 15-year period (1986-2000), a total of 1,650 children with culture proven typhoid fever were seen at AKUMC with a documented follow-up period of $\geq 8$ weeks. Of these, 1,627 had their entire treatment course recorded. Baseline characteristics for the cohort are provided in Table 1. All recorded variables were equally distributed among relapsed and non-relapsed patients (data not shown) with the exception that those receiving partial or complete therapy with second-line antimicrobials were protected against relapse (Table 2). We experienced an increase in relapse rates starting in the early 1990s concurrent with the emergence of MDR strains (Figure 1). A total of 949 patients $(57.5 \%)$ had infection with pan-sensitive strains of enteric fever and 543 patients $(32.9 \%)$ with MDR strains of enteric fever. The remainder were infected with strains displaying partial drug resistance to either one or two of the aforementioned antimicrobials. Subgroup analysis of MDR typhoid fever cases revealed that the rate of relapse was higher in those initially presenting with rather than without constipation $(15 \%$ versus $4.4 \%, \mathrm{p}=0.01)$ and in those presenting within 14 days of fever onset $(6.4 \%$ versus $0.8 \%, \mathrm{p}=$ 0.01). Patients with MDR enteric fever had a lower relapse rate when treated with quinolones or cephalosporins (Table 2), but this was not true for pan-sensitive or partially drug resistant strains of Salmonella. Logistic regression analysis for patients infected with MDR strains found presentation within 14 days of fever onset and constipation continue to be variables associated with relapse $(p=0.035$ and $p=$ 0.003 respectively) whereas treatment with quinolones or cephalosporins during the treatment course continue to be associated with decreased relapse $(\mathrm{p}=0.01)$.

In patients infected with pan-sensitive strains of enteric fever, treatment with quinolones or cephalosporins did not significantly alter the relapse rate. In this subgroup of patients, relapse was lower in those presenting with rather than without diarrhoea (3.7\% vs. $9.6 \%, \mathrm{p}=0.001)$. 
Table 1. Clinical characteristics of the cohort

\begin{tabular}{|c|c|}
\hline Clinical characteristic & $\begin{array}{l}\text { Total } n=1650 \\
n(\% \text { of cases })\end{array}$ \\
\hline Clinical toxicity & $428(32.0)$ \\
\hline Diarrhoea & $612(37.1)$ \\
\hline Constipation & $143(8.7)$ \\
\hline Abdominal Pain & $658(39.9)$ \\
\hline Abdominal Tenderness & $385(23.3)$ \\
\hline Hepatomegaly & $657(39.8)$ \\
\hline Splenomegaly & $305(18.5)$ \\
\hline Pallor & $344(20.8)$ \\
\hline Jaundice & $31(1.9)$ \\
\hline Cough & $224(13.6)$ \\
\hline Obtundation & $91(5.5)$ \\
\hline Hospital Admission & $981(59.5)$ \\
\hline Infection with MDR strain(s) & $543(32.9)$ \\
\hline Infection with $S$. Typhi strain(s) & $1459(88.4)$ \\
\hline Subsequent relapse & $108(6.5)$ \\
\hline & mean $(\mathrm{SD})$ \\
\hline Age (years) & $6.2(3.9)$ \\
\hline Weight (kg) & $18.3(10.0)$ \\
\hline Days of illness at presentation & $9.6(9.8)$ \\
\hline Hemoglobin (mg/dL) & $10.9(1.7)$ \\
\hline White Blood Cell Count $\left(10^{3} / \mu \mathrm{L}\right)$ & $9316(5256)$ \\
\hline
\end{tabular}

\section{Discussion}

In this report we describe our experience regarding enteric fever relapse spanning 15 years and 1,650 children during the emergence of multiple-drug resistant strains in South Asia. The relapse rate reported in our study is consistent with that described in the literature. Though the advent of antibiotics has considerably reduced morbidity and mortality associated with enteric fever, the relapse rate remains at $5 \%$ to $10 \%$ in immunocompetent hosts [2,7]. Factors influencing the efficacy of antimicrobials in decreasing the relapse rate include bactericidal effectiveness, the ability to permeate the intracellular environment, as well as the appropriate dose and duration of therapy [8]. Fluoroquinolones and broadspectrum cephalosporins have a better treatment profile in this regard [9].

To our surprise, when examining the entire cohort, we did not find any clinical or laboratory variables predictive of future relapse. This may be due to the emergence of MDR strains of enteric fever during the study period leading to two clinically distinct populations of patients within the cohort. When receiving partial courses or full therapy with fluoroquinolones the difference in relapse rates between MDR and pan-sensitive typhoid strains were not statistically significant. As may be expected, however, patients with drug resistant typhoid who received ineffective therapy had a relapse rate almost twice that of those infected with pan-sensitive strains (Table 2).

We found diarrhoea to be associated with lower relapse rates in children infected with pan-sensitive enteric fever whereas those infected with MDR strains had a higher relapse rate when presenting with constipation or starting specific therapy within 14 days of fever onset. Perhaps of greater clinical significance, the use of quinolones or cephalosporins as part of the treatment course protected against subsequent relapse. In those areas where MDR typhoid caused by $S$. Typhi is prevalent, it would be prudent to consider empirical treatment of patients with a cephalosporin or quinolone until infection is 
Table 2. Relapse rate categorised by bacterial drug resistance and antimicrobials used

\begin{tabular}{|l|c|c|c|}
\cline { 2 - 4 } \multicolumn{1}{c|}{} & $\begin{array}{c}\text { Initial therapy of exclusively } \\
\text { ampicillin, chloramphenicol, } \\
\text { or TMP-SMX }\end{array}$ & $\begin{array}{c}\text { Partial or full Initial therapy } \\
\text { with cephalosporins or } \\
\text { quinolones }\end{array}$ & \multicolumn{1}{c|}{ p value } \\
\hline Organism resistance profile & \multicolumn{2}{|c|}{ Cases of relapse / total cases (percent) } & 0.32 \\
\hline Pan-sensitive & $47 / 559(8.4)$ & $25 / 377(6.6)$ & $1^{\mathrm{a}}$ \\
\hline Partial drug resistance & $3 / 71(4.2)$ & $4 / 86(4.7)$ & $0.018^{\mathrm{a}}$ \\
\hline Multi-drug resistance & $5 / 31(16.1)$ & $23 / 506(4.5)$ & 0.018 \\
\hline All patients & $55 / 661(8.3)$ & $52 / 969(5.4)$ & \multicolumn{2}{c}{} \\
\hline
\end{tabular}

proven to be caused by a drug sensitive strain. This is now the standard treatment strategy in our institution and in our experience in much of South Asia.

Although the reasons for our findings are unclear, one unifying possibility is that the clinical factors noted above may modify the load of Salmonella within the body and reticuloendothelial system (RES). Treatment of MDR enteric fever with quinolones may be predicted to better eradicate Salmonella from the RES than treatment with firstline antimicrobials. Infection for greater than 14 days prior to therapy may lead to a greater immune system activation thereby allowing improved Salmonella clearance from the RES. Likewise, as this organism is shed through the stool, it is possible that constipation may lead to a higher load of organism within the body whereas diarrhoea could be predicted to cause the opposite effect.

Interpretation of our data may be limited as we report a single institutional experience rather than surveillance of a large population. However, the age distribution in our study is similar to that of population based studies in India and $49 \%$ of patients were treated entirely as outpatients. Although we defined relapse clinically and by identical antibiogram, more sophisticated techniques, including pulsed field gel electrophoresis (PFGE), have since become available to determine strain differences. We would expect however that use of PFGE to detect relapse rather than antibiogram would lead to a clearer classification of patients with new infections compared to relapsed cases. If this reinfection occurred in significant numbers however we expect use of the antibiogram would mask differences between the groups rather than lead to an increase in differences. No MIC data is available from this time period; thus more subtle differences in MIC among "drug-sensitive" strains are not presented. It is of note that the study period was prior to the emergence of widespread quinolone resistance in many parts of the world [10].
The strength of this study is that we report the largest known cohort of patients to examine the risk factors for enteric fever relapse in children. This large cohort size allows for the robust subgroup analysis from which we report significant findings. Our institution has followed a standardized clinical treatment algorithm over many years which has allowed consistency in treatment regimens. Additionally, the study period encompasses the era during which MDR enteric fever emerged in South Asia, allowing direct comparison of drug-sensitive and MDR strains.

In conclusion, we report clinical variables in patients infected with both drug-sensitive and MDR enteric fever that correlate with relapse after a clinical cure has been achieved. These risk factors will be of value in creating treatment and follow-up algorithms for this large patient population.

\section{References}

1. Crump JA, Luby SP, Mintz ED (2004) The global burden of typhoid fever. Bull World Health Organ 82: 346-353.

2. Braunwald E (2001) Harrison's principles of internal medicine. 15th ed. New York: McGraw-Hill, Medical Pub. Division, $2958 \mathrm{p}$.

3. Viret J and Cryz S. (1995) Protective immunity induced by typhoid fever and vaccination. Southeast Asian J Trop Med Public Health 26: 150-159.

4. Bhutta ZA, Naqvi SH, Razzaq RA, Farooqui BJ (1991) Multidrug-resistant typhoid in children: presentation and clinical features. Rev Infect Dis 13: 832-836.

5. Rowe B, Ward LR, Threlfall EJ (1990) Spread of multiresistant Salmonella typhi. Lancet 336: 1990.

6. Bauer AW, Kirby WM, Sherris JC, Turck M (1966) Antibiotic susceptibility testing by a standardized single disk method. Am J Clin Pathol 45: 493-496.

7. Bhutta ZA (1996) Therapeutic aspects of typhoidal salmonellosis in childhood: the Karachi experience. (1996) Ann Trop Paediatr 16: 299-306.

8. Gotuzzo E, Morris JG, Jr., Benavente L, Wood PK, Levine O, Black RE, Levine MM (1987) Association between specific plasmids and relapse in typhoid fever. J Clin Microbiol 25: 1779-1781.

9. Andriole VT (1998) The Quinolones. New York: Academic. (author: please supply page numbers per author guidelines on the JIDC web site. Thank you. 
10. Murdoch DA, Banatvaia N, Bone A, Shoismatulloev BI, Ward LR, Threlfall EJ (1998) Epidemic ciprofloxacinresistant Salmonella typhi in Tajikistan. Lancet 351: 339.

\section{Corresponding author}

Dr. Zulfiqar A. Bhutta

M.B., B.S., F.R.C.P., F.R.C.P.C.H., Ph.D.

Department of Pediatrics

The Aga Khan University Hospital

PO Box 3500, Stadium Road

Karachi, 74800, Pakistan

Telephone: 92-21-493-0051 ext. 4729, 4727

Fax: 92-21-493-4294

Email: zulfiqar.bhutta@aku.edu

Conflict of interests: No conflict of interests is declared. 